\title{
Steady-State Thermoelastic Analytical Solutions for Insulated Pipelines
}

\author{
M. Fraldi, ${ }^{1,2}$ L. Esposito, ${ }^{1}$ F. Carannante, ${ }^{3}$ A. Cutolo, ${ }^{4}$ and L. Nunziante ${ }^{1}$ \\ ${ }^{1}$ Department of Structures for Engineering and Architecture, University of Napoli Federico II, Napoli, Italy \\ ${ }^{2}$ Interdisciplinary Research Center for Biomaterials, University of Napoli Federico II, Napoli, Italy \\ ${ }^{3}$ Department of Fire Watch, Public Rescue and Civil Protection, Italian Ministry of Interior, Roma, Italy \\ ${ }^{4}$ Department of Chemical, Materials and Production Engineering, University of Napoli Federico II, Napoli, Italy
}

Correspondence should be addressed to M. Fraldi; fraldi@unina.it

Received 3 May 2016; Revised 9 August 2016; Accepted 18 September 2016

Academic Editor: Francesco Pellicano

Copyright (c) 2016 M. Fraldi et al. This is an open access article distributed under the Creative Commons Attribution License, which permits unrestricted use, distribution, and reproduction in any medium, provided the original work is properly cited.

A steady-state thermoelastic analytical solution for a multilayer hollow cylinder, composed of an arbitrary number of phases and subject to both radial pressure and temperature gradient, is presented. By assuming each phase to be homogeneous and thermally isotropic and by varying the mechanical and thermal constitutive parameters, a sensitivity analysis has been performed with the aim of finally applying the study to the mechanical behaviour of an industrial pipeline composed of three phases (steel, insulating coating, and polyethylene) under the action of the above-mentioned load conditions. By making reference to a classical Henckyvon Mises criterion, the stress profiles along the thickness of the layers have been carried out, also localizing the onset of plasticity as a function of the temperature variations, material properties, and geometrical features characterizing the composite structure of interest. At the end, some numerical results of practical interest in the engineering applications have been specialized to three different insulated coating materials (expanded polyurethane, laminate glass, and syntactic foam), to highlight the cases in which thermal properties and loads can significantly interfere with the mechanical response in pipes, in terms of stresses, in this way suggesting possible strategies for avoiding unexpected failure and supporting the optimal structural design of these systems.

\section{Introduction}

In the last years, a growing demand for insulated pipes operating at higher temperatures has addressed a need of insulation materials able to withstand thermal degradation and physical stress. As a consequence, the design of piping, with particular attention on the thermomechanical properties of the adopted materials, has become an important aspect in any energy consuming system, due to the considerable impact of the insulation arrangement and sizing on the overall performance of the structure. Actually, the design of pipelines is usually based on either worked before or educated estimates and is deeply influenced by both hardware and energy costs. Oversized pipes are designed to preserve thermal energy, and adequate insulations are chosen to achieve both low thermal conductivity and inertia, in order to reduce heat loss from hot surfaces and heat gain in refrigeration systems. In fact, either inadequate thickness or deterioration of existing insulation causes high energy consumptions, so that retrofitting or reinsulation techniques, strictly related to the development of higher performances at lower prices insulating materials, are becoming a very attractive energy saving method. In this framework, multilayer insulated pipes could be a suitable alternative, in which different materials can be used to enhance energetic performances of pipelines. De facto, multilayered insulated pipelines can be considered as laminate composites. Laminate composites and socalled functionally graded material structures (FGMs) have been deeply studied thanks to their capability of working in high-temperature environments and many research works have been carried out for characterizing spherical, plated, and cylindrical shapes FGMs by means of both analytical and numerical approaches [1-7].

In particular, by adopting an analytical approach, different scientific studies have been devoted to investigate 
the effects of nonlinear constitutive properties and nonhomogeneities on the response of cylindrical FGMs subject to nonlinear distribution of temperature along the radius. Eraslan [8] developed the analytical solutions of nonuniform heat-generating composite tubes with fixed ends, subject to thermally induced axisymmetric elastoplastic deformations, calculating the results as a consequence of nonlinearly hardening properties. Instead, Shao [9] derived analytical solutions of a circular hollow cylinder composed of different homogeneous fictitious layers in the radial direction, finding mechanical and thermal stresses for the two-dimensional thermoelastic problems. Fraldi et al. [10] showed the exact analytical solutions for the elastic response of a solid circular cylinder composed of the assembly of a central core and $n$ surrounding hollow phases, all made of different homogeneous elastic materials; under de Saint Venant-like load conditions, they produced overall elastic relationships between generalized stresses and strains in cases of axial force, torque, bending, and shear, also registering the effects of Poisson ratios on the overall stiffness. Moreover, Fraldi et al. [11], in hypothesis of no-decaying of selected mechanical quantities, obtained a special form of the biharmonic Love's function, as expression of the radius and the coordinate along the axis. Eraslan and Akis [3], in the framework of small deformation theory, obtained plane strain analytical solutions for a functionally graded elastic-plastic pressurized tube, making reference to Tresca's yield criterion, showing that the elastoplastic response of the pressurized tube is significantly affected by the material nonhomogeneity. By adopting Green's function approach, Kim and Noda [6] solved the twodimensional unsteady temperature problem, determining the associated thermal stresses in an infinite hollow cylinder with radially dependent properties. Further studies by Jabbari et al. [7] derived analytical solutions for one-dimensional steady-state thermoelastic circular hollow thick cylinders and, by assuming the temperature distribution and material properties to be functions of the radius with a power law distribution, obtained the solution of the heat conduction and Navier equation, while Liew et al. [12] determined analytical solutions of a circular hollow cylinder by a novel limiting process employing the solutions of homogeneous circular hollow cylinders. Also, several studies have focused the attention on the analysis of composite pipelines under combined thermal and mechanical conditions, with the aim of highlighting both specific aspects of interest for practical engineering applications [13-16] and theoretical insights [1719].

In this paper, by using a multilayered approach based on the theory of laminated composites, a steady-state thermoelastic analytical solution for a multilayer hollow cylinder, composed of an arbitrary number of phases and subject to both radial pressure and temperature gradients, is proposed. Successively, by assuming the material properties to be piecewise radially varying (homogeneous in each layer) and temperature-independent, parametric analyses and analytical solutions for a three-layered hollow cylinder composed of steel, insulating coating, and polyethylene jacket are presented. The results are shown by varying Young's moduli, Poisson's ratios, thermal conductivity, and linear expansion coefficients of the constituent materials. In order to localize the onset of plasticity and following the Hencky-von Mises criterion, the maximum equivalent stress has been finally determined both in the steel phase and in the insulate coating. At the end, a numerical example is reported considering the insulate coating composed of expanded polyurethane, laminate glass, and syntactic foam.

\section{Basic Equations for the Steady-State Problem}

A hollow multilayered cylinder constituted by $n$ phases with finite length $L$ has been considered (Figure 1(a)). The external and internal radii of the multilayer cylinder and the radius at interface between the generic $i$ th and $(i+1)$ th phases have been denoted by $R^{(n)}, R^{(0)}$, and $R^{(i)}$, respectively. The mechanical and thermal properties of each layer have been assumed to be homogeneous and isotropic and are denoted with apex (i). A thermal gradient has been considered across the pipe walls, with external and internal values of, respectively, $T_{\text {ext }}$ and $T_{\text {int }}$, and constant pressure values have been applied both on the outer $\left(p_{\text {ext }}\right)$ and on the inner surfaces $\left(p_{\text {int }}\right)$, that is, at $r=R^{(n)}$ and $r=R^{(0)}$, respectively.

By assuming a cylindrical coordinates system $\{r, \theta, z\}$, if the hollow cylinder is subjected to radial temperature variation and radial uniform pressure, one can seek the displacement as reduced to the sole radial and axial (along $z$-direction) components in the following form, the temperature becoming a function of the sole variable $r$ at each $i$ th layer, so that

$$
\begin{aligned}
& u_{r}^{(i)}=u_{r}^{(i)}(r), \\
& u_{z}^{(i)}=\varepsilon_{0} z, \\
& T^{(i)}=T^{(i)}(r),
\end{aligned}
$$

$$
\forall i \in\{1,2, \ldots, n\}
$$

where apexes $1,2, \ldots, n$ denote the pipe layers and $\varepsilon_{0}$ represents the constant axial strain shared among the phases [11]. Therefore, the nonvanishing strain components take the form

$$
\begin{aligned}
\varepsilon_{r r}^{(i)} & =u_{r, r}^{(i)}, \\
\varepsilon_{\theta \theta}^{(i)} & =r^{-1} u_{r}^{(i)}, \\
\varepsilon_{z z}^{(i)} & =\varepsilon_{0},
\end{aligned}
$$

$\forall i \in\{1,2, \ldots, n\}$.

Similarly, considering each phase as isotropic, the nonvanishing stress components can be written as

$$
\begin{aligned}
\sigma_{r r}^{(i)}= & 2 \mu^{(i)} u_{r, r}^{(i)}+\lambda^{(i)}\left(u_{r, r}^{(i)}+r^{-1} u_{r}^{(i)}+\varepsilon_{0}\right) \\
& -\left(3 \lambda^{(i)}+2 \mu^{(i)}\right) \alpha^{(i)}\left(T^{(i)}-T_{R}\right), \\
\sigma_{\theta \theta}^{(i)}= & 2 \mu^{(i)} r^{-1} u_{r}^{(i)}+\lambda^{(i)}\left(u_{r, r}^{(i)}+r^{-1} u_{r}^{(i)}+\varepsilon_{0}\right) \\
& -\left(3 \lambda^{(i)}+2 \mu^{(i)}\right) \alpha^{(i)}\left(T^{(i)}-T_{R}\right),
\end{aligned}
$$




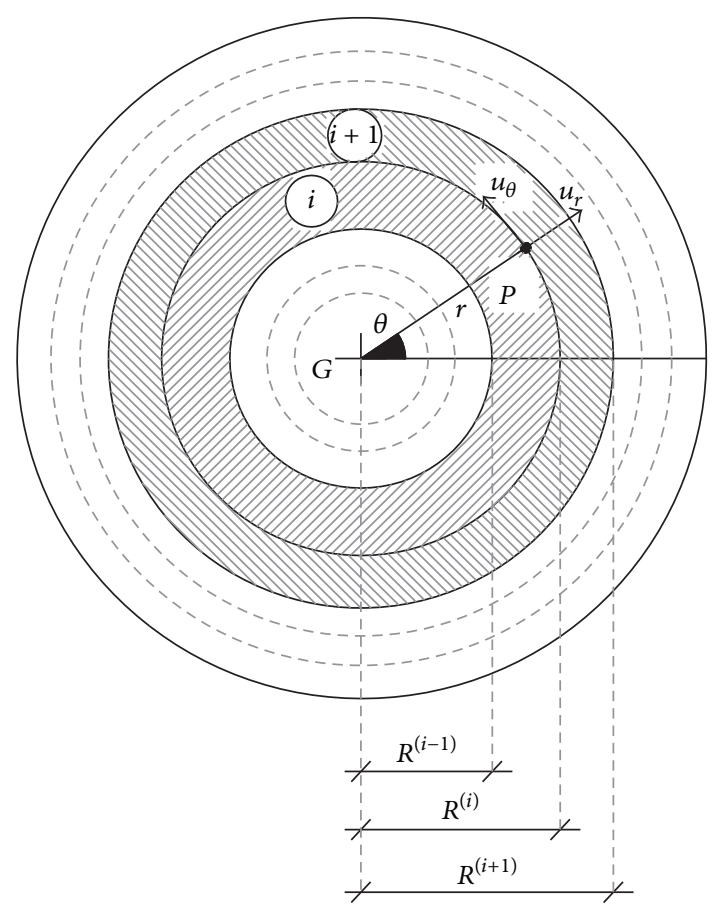

(a)

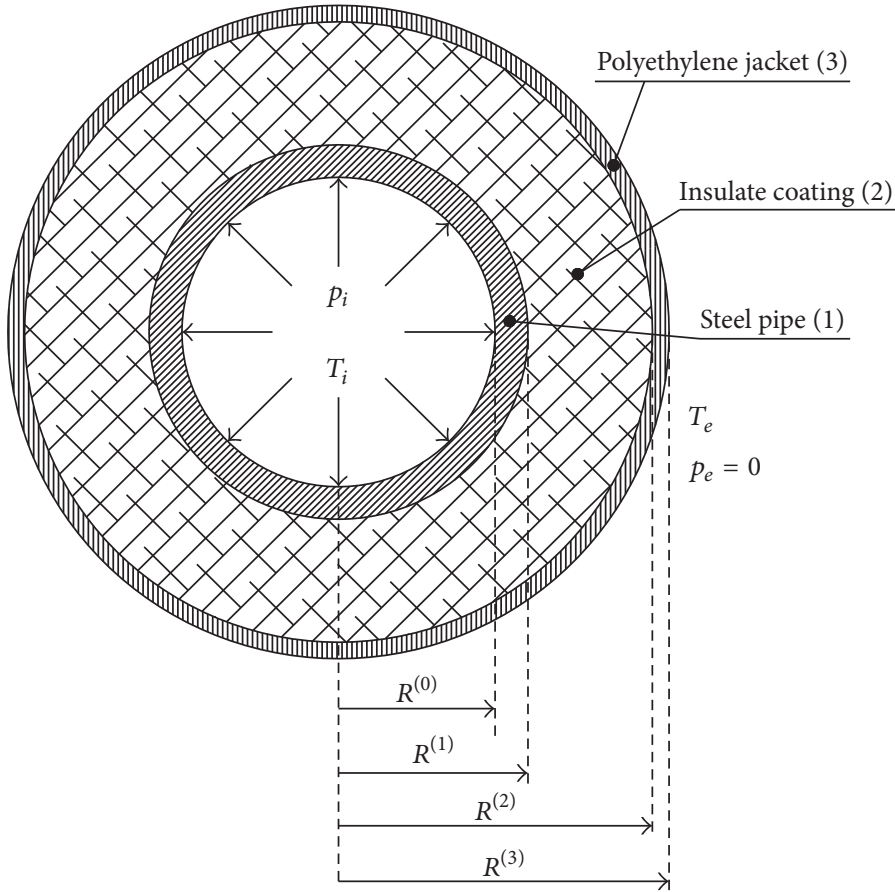

(b)

FIGURE 1: (a) Multilayer cylinder composed of $n$-hollow cylinders; (b) cross section of an insulated pipeline composed of three phases: steel (1), insulate coating (2), and polyethylene jacket (3).

$$
\begin{array}{r}
\sigma_{z z}^{(i)}=2 \mu^{(i)} \varepsilon_{0}+\lambda^{(i)}\left(u_{r, r}^{(i)}+r^{-1} u_{r}^{(i)}+\varepsilon_{0}\right) \\
-\left(3 \lambda^{(i)}+2 \mu^{(i)}\right) \alpha^{(i)}\left(T^{(i)}-T_{R}\right), \\
\forall i \in\{1,2, \ldots, n\},
\end{array}
$$

where $\mu^{(i)}, \lambda^{(i)}, \alpha^{(i)}$, and $T^{(i)}$ are the two Lamé elastic moduli, the thermoelastic coefficients, and temperature field for the $i$ th layer, respectively.

By applying the hypothesis of axial-symmetric and in absence of body forces, the equilibrium equations reduce to the sole nontrivial one:

$$
\sigma_{r r, r}^{(i)}+r^{-1}\left(\sigma_{r r}^{(i)}-\sigma_{\theta \theta}^{(i)}\right)=0 \quad \forall i \in\{1,2, \ldots, n\}
$$

By substituting the strain-displacement relations (2) in the stress-strain relations (3) and thus in the equilibrium equation (4), the displacements in each phase become the unknown functions to be determined. If the Lamé coefficients are expressed as functions of elastic moduli $E^{(i)}$ and $v^{(i)}$, by invoking the well-known following relations,

$$
\begin{aligned}
\mu^{(i)} & =\frac{E^{(i)}}{2\left(1+\nu^{(i)}\right)}, \\
\lambda^{(i)} & =\frac{E^{(i)} \nu^{(i)}}{\left[\left(1+\nu^{(i)}\right)\left(1-2 \nu^{(i)}\right)\right]},
\end{aligned}
$$

the displacements formulation of the equilibrium equations allows reducing the problem at hand to one ordinary differential equation:

$$
\left[r^{-1}\left(r u_{r}^{(i)}\right)_{, r}\right]_{, r}=2 \zeta^{(i)} T_{, r}^{(i)}, \quad \forall i \in\{1,2, \ldots, n\}
$$

where the constant $\zeta^{(i)}=\left[\alpha^{(i)}\left(1+v^{(i)}\right)\right] /\left[2\left(1-v^{(i)}\right)\right]$.

In order to impose both equilibrium and compatibility at the boundary surfaces between two generic adjacent phases, under the hypothesis of perfect bond at the cylindrical interfacial boundaries (no delamination or friction phenomena), the generic solution for a functionally graded circular cylinder constituted by $n$ arbitrary hollow phases can be set. In particular, the following boundary conditions have been established in terms of displacements at the interfaces

$$
\begin{aligned}
& u_{r}^{(i)}\left(r=R^{(i)}\right)=u_{r}^{(i+1)}\left(r=R^{(i+1)}\right) \\
& \forall i \in\{1,2, \ldots, n-1\}
\end{aligned}
$$

and the equilibrium of stresses at the generic interface requires having

$$
\begin{aligned}
& \sigma_{r r}^{(i)}\left(r=R^{(i)}\right)=\sigma_{r r}^{(i+1)}\left(r=R^{(i+1)}\right) \\
& \forall i \in\{1,2, \ldots, n-1\} .
\end{aligned}
$$


Also, equilibrium equations for the tractions at the inner and the outer cylindrical boundary surfaces are given by

$$
\begin{aligned}
& \sigma_{r r}^{(0)}\left(r=R^{(0)}\right)=p_{\text {int }}, \\
& \sigma_{r r}^{(n)}\left(r=R^{(n)}\right)=p_{\text {ext }},
\end{aligned}
$$

where $p_{\text {ext }}$ and $p_{\text {int }}$ are the pressure applied on the inner and the outer surfaces of the multilayered cylinder, respectively.

It is worth highlighting that the choice to assume a displacement field in which the axial strain $\varepsilon_{0}$ is constant and common to all the phases automatically guarantees the compatibility of the displacement along the $z$-direction at the interfaces, also ensuring vanishing antiplane shear stresses $\sigma_{r z}^{(i)}$ and, by virtue of the particular form of the other displacement functions, torsionless deformation and stress regimes; that is, $\sigma_{\vartheta z}^{(i)}=0$. As a consequence of this choice for the axial strain, two possibilities can be taken into account, say first in which $\varepsilon_{0}=0$ and another (complementary) one in which, dually, the axial resultant $F$ emerging from any overall cylinder cross section along the $z$-direction is set to be equal to zero. In the first case that is the case considered in the present work the problem reduces to plane strain and the resultant $F$ will be identified as the reaction that allows the deformation to remain plane through different values of stresses $\sigma_{z z}^{(i)}$ generated within each phase. On the contrary, the same general form assumed above for the displacements could be exploited to replicate the dual case in which the axial elongation (or contraction) $\varepsilon_{0}$ is chosen to give $F=0$.

As anticipated above, without loss of generality with respect to the possibility of reproducing both the cases described above, in what follows the condition $\varepsilon_{0}=0$ is set and hence an axial force $F$, whose magnitude does not depend on $z$, can be found once the solutions in terms of displacements and related stresses will be completely defined; that is,

$$
\begin{aligned}
F & =\sum_{i=1}^{n} \int_{0}^{2 \pi} \int_{R^{(i-1)}}^{R^{(i)}} \sigma_{z z}^{(i)}(r) r d r d \theta \\
& =2 \pi \sum_{i=1}^{n} \int_{R^{(i-1)}}^{R^{(i)}} \sigma_{z z}^{(i)}(r) r d r .
\end{aligned}
$$

Hypothesizing axial-symmetric load conditions for each phase, the steady-state conduction equation can be written as

$$
T_{, r r}^{(i)}+r^{-1} T_{, r}^{(i)}=0 \quad \forall i \in\{1,2, \ldots, n\} .
$$

At the interfaces between the phases, the temperature and heat flux are equal, so that the following conditions can be written:

$$
\begin{aligned}
T^{(i)}\left(r=R^{(i)}\right) & =T^{(i+1)}\left(r=R^{(i)}\right) \\
k^{(i)} T_{, r}^{(i)}\left(r=R^{(i)}\right)=k^{(i+1)} T_{, r}^{(i+1)} & \left(r=R^{(i)}\right) \\
& \forall i \in\{0,1, \ldots, n-1\},
\end{aligned}
$$

where $k^{(i)}$ is the thermal conductivity coefficient of the $i$ phase.

Similarly, on the inner and the outer surfaces, the temperature is equal to $T_{\text {ext }}$ and $T_{\text {int }}$, respectively,

$$
\begin{aligned}
& T^{(n)}\left(r=R^{(n)}\right)=T_{\text {ext }} \\
& T^{(0)}\left(r=R^{(0)}\right)=T_{\text {int }} .
\end{aligned}
$$

\section{Analytical Solution in Closed-Form for Multilayered Cylinder in Plane Strain Subject to Radial Temperature Variation and Uniform Pressure}

By integrating (6), the displacements solution in each phase of a multilayered cylinder can be found in closed-form as follows:

$$
\begin{aligned}
u_{r}^{(i)}=2 \zeta^{(i)} r^{-1} \int r T^{(i)}(r) d r+C_{1}^{(i)} r+ & C_{2}^{(i)} r^{-1} \\
& \forall i \in\{1,2, \ldots, n\},
\end{aligned}
$$

where $C_{1}^{(i)}$ and $C_{2}^{(i)}$ are integration constants to be determined. By integrating Fourier's equation reported in (11), the temperature function for the steady-state problem in each phase takes the form

$$
T^{(i)}(r)=A_{1}^{(i)} \log r+A_{2}^{(i)} \quad \forall i \in\{1,2, \ldots, n\},
$$

where $A_{1}^{(i)}$ and $A_{2}^{(i)}$ are parameters to be specialized through the boundary conditions. Thus, the heat flux in each phase is given by

$$
q^{(i)}=-k^{(i)} T_{, r}^{(i)}=-k^{(i)} A_{1}^{(i)} r^{-1} \quad \forall i \in\{1,2, \ldots, n\},
$$

and substituting the temperature function (15) in (14), the explicit expression of the displacement function can be obtained:

$$
\begin{array}{r}
u_{r}^{(i)}=C_{1}^{(i)} r+C_{2}^{(i)} r^{-1}+\zeta^{(i)} r\left[A_{1}^{(i)}\left(\log r-\frac{1}{2}\right)+A_{2}^{(i)}\right] \\
\forall i \in\{1,2, \ldots, n\}
\end{array}
$$

By applying the strain-displacement relationship, the not vanishing strain components can be in turn determined:

$$
\begin{aligned}
& \varepsilon_{r r}^{(i)}=C_{1}^{(i)}-C_{2}^{(i)} r^{-2}+\zeta^{(i)}\left[A_{1}^{(i)}\left(\log r+\frac{1}{2}\right)+A_{2}^{(i)}\right] \\
& \varepsilon_{\theta \theta}^{(i)}=C_{1}^{(i)}+C_{2}^{(i)} r^{-2}+\zeta^{(i)}\left[A_{1}^{(i)}\left(\log r-\frac{1}{2}\right)+A_{2}^{(i)}\right]
\end{aligned}
$$


and, by applying the stress-strain relationship, the nonzero stress components can be explicitly written as

$$
\begin{aligned}
\sigma_{r r}^{(i)} & =\frac{E^{(i)}}{1+\nu^{(i)}}\left\{\frac{C_{1}^{(i)}}{1-2 \gamma^{(i)}}-C_{2}^{(i)} r^{-2}\right. \\
& \left.-\zeta^{(i)}\left[A_{1}^{(i)}\left(\log r-\frac{1}{2}\right)+A_{2}^{(i)}\right]\right\}+\Omega^{(i)} T_{R}, \\
\sigma_{\theta \theta}^{(i)} & =\frac{E^{(i)}}{1+\nu^{(i)}}\left\{\frac{C_{1}^{(i)}}{1-2 \gamma^{(i)}}+C_{2}^{(i)} r^{-2}\right. \\
& \left.-\zeta^{(i)}\left[A_{1}^{(i)}\left(\log r+\frac{1}{2}\right)+A_{2}^{(i)}\right]\right\}+\Omega^{(i)} T_{R}, \\
\sigma_{z z}^{(i)} & =\frac{E^{(i)}}{1+\nu^{(i)}}\left\{\frac{2 C_{1}^{(i)} \nu^{(i)}}{1-2 \nu^{(i)}}-2 \zeta^{(i)}\left(A_{1}^{(i)} \log r+A_{2}^{(i)}\right)\right\}
\end{aligned}
$$

$$
+\Omega^{(i)} T_{R}
$$

$\forall i \in\{1,2, \ldots, n\}$

where $\Omega^{(i)}=\alpha^{(i)} E^{(i)} /\left(1-2 v^{(i)}\right)$.

For planar isotropy and nonhomogeneity in radial direction, the equivalent stress, based on the Hencky-von Mises criterion, is given by

$$
\begin{aligned}
\sigma_{\mathrm{eq}}^{(i)} & =2^{-1 / 2}\left[\left(\sigma_{r r}^{(i)}-\sigma_{\theta \theta}^{(i)}\right)^{2}+\left(\sigma_{r r}^{(i)}-\sigma_{z z}^{(i)}\right)^{2}\right. \\
& \left.+\left(\sigma_{\theta \theta}^{(i)}-\sigma_{z z}^{(i)}\right)^{2}\right]^{1 / 2} .
\end{aligned}
$$

By substituting (19) in (20), the equivalent stress is given by

$$
\begin{aligned}
\sigma_{\mathrm{eq}}^{(i)} & =\frac{E^{(i)}}{1+\nu^{(i)}} \\
& \cdot \sqrt{C_{1}^{(i) 2}+3 C_{2}^{(i) 2} r^{-4}+\zeta^{(i)}\left[2 C_{1}^{(i)}\left(A_{1}^{(i)} \log r+A_{2}^{(i)}\right)-3 A_{1}^{(i)} C_{2}^{(i)} r^{-2}\right]+\zeta^{(i) 2}\left[A_{2}^{(i) 2}+2 A_{1}^{(i)} A_{2}^{(i)} \log r+A_{1}^{(i) 2}\left(\frac{3}{4}+\log ^{2} r\right)\right]} .
\end{aligned}
$$

Finally, by utilizing the expressions of the temperature, heat flux, radial displacement, and radial stress in each phase, the boundary conditions for the multilayered cylinder can be yet written explicitly. Then, the compatibility boundary conditions (7) become

$$
\begin{aligned}
& C_{1}^{(i+1)}-C_{1}^{(i)}+\left(C_{2}^{(i+1)}-C_{2}^{(i)}\right) R^{(i)-2} \\
&=\zeta^{(i)}\left[A_{1}^{(i)}\left(\log R^{(i)}-\frac{1}{2}\right)+A_{2}^{(i)}\right] \\
&-\zeta^{(i+1)}\left[A_{1}^{(i+1)}\left(\log R^{(i)}-\frac{1}{2}\right)+A_{2}^{(i+1)}\right] \\
& \forall i \in\{1,2, \ldots,(n-1)\} .
\end{aligned}
$$

The equilibrium conditions (8) at interfaces are given by

$$
\begin{aligned}
& \frac{E^{(i+1)}}{\left(1+\nu^{(i+1)}\right)}\left[\frac{C_{2}^{(i+1)}}{R^{(i) 2}}-\frac{C_{1}^{(i+1)}}{1-2 \nu^{(i+1)}}\right] \\
& -\frac{E^{(i)}}{\left(1+\nu^{(i)}\right)}\left[\frac{C_{2}^{(i)}}{R^{(i) 2}}-\frac{C_{1}^{(i)}}{1-2 \nu^{(i)}}\right] \\
& =T_{R}\left(\Omega^{(i+1)}-\Omega^{(i)}\right) \\
& +\psi^{(i)}\left[A_{2}^{(i)}+A_{1}^{(i)}\left(\log R^{(i)}-\frac{1}{2}\right)\right] \\
& -\psi^{(i+1)}\left[A_{2}^{(i+1)}+A_{1}^{(i+1)}\left(\log R^{(i)}-\frac{1}{2}\right)\right] \\
& \quad \forall i \in\{1,2, \ldots,(n-1)\},
\end{aligned}
$$

where $\psi^{(i)}=(1 / 2)\left[\left(\alpha^{(i)} E^{(i)}\right) /\left(1-v^{(i)}\right)\right]$.
The equilibrium equations on the external and internal surface of the solid (9) also give

$$
\begin{aligned}
& \frac{E^{(1)}}{1+\nu^{(1)}}\left(\frac{C_{1}^{(1)}}{1-2 \gamma^{(1)}}-\frac{C_{2}^{(1)}}{R^{(0) 2}}\right) \\
& =-\Omega^{(1)} T_{R}+\psi^{(1)}\left[A_{1}^{(1)}\left(\log R^{(0)}-\frac{1}{2}\right)+A_{2}^{(1)}\right] \\
& \quad+p_{\text {int }} \\
& \frac{E^{(n)}}{1+\nu^{(n)}}\left(\frac{C_{1}^{(n)}}{1-2 \gamma^{(n)}}-\frac{C_{2}^{(n)}}{R^{(n) 2}}\right) \\
& =-\Omega^{(n)} T_{R}+\psi^{(n)}\left[A_{1}^{(n)}\left(\log R^{(n)}-\frac{1}{2}\right)+A_{2}^{(n)}\right] \\
& \quad+p_{\text {ext }} .
\end{aligned}
$$

The temperature and flux continuity at the interfaces between the adjacent phases (12) furnish the following relations:

$$
\begin{aligned}
& {\left[A_{1}^{(i+1)}-A_{1}^{(i)}\right] \log R^{(i)}+A_{2}^{(i+1)}-A_{2}^{(i)} }=0, \\
& k^{(i+1)} A_{2}^{(i+1)}-k^{(i)} A_{2}^{(i)}=0, \\
& \forall i \in\{1,2, \ldots,(n-1)\},
\end{aligned}
$$

to be associated with the boundary conditions on the external and the internal surfaces, related to the temperature (13); that is,

$$
\begin{aligned}
& A_{1}^{(1)} \log R^{(0)}+A_{2}^{(1)}=T_{\mathrm{int}}, \\
& A_{1}^{(n)} \log R^{(n)}+A_{2}^{(n)}=T_{\mathrm{ext}} .
\end{aligned}
$$


Equations (22)-(26) thus represent an algebraic system constituted by $4 \times n$ equations in the $4 \times n$ unknown parameters reported below:

$$
A_{1}^{(i)}, A_{2}^{(i)}, C_{1}^{(i)}, C_{2}^{(i)} \quad \forall i \in\{1,2, \ldots, n\}
$$

The algebraic system mentioned above can be conveniently rearranged by subdividing it into two uncoupled algebraic systems:

(i) The first one, constituted by (25) and (26) and characterized by $2 \times n$ equations and $2 \times n$ unknown parameters $A_{1}^{(i)}, A_{2}^{(i)}, \forall i \in\{1,2, \ldots, n\}$.

(ii) The second one, composed of (22), (23), and (24) and characterized by $2 \times n$ equations and $2 \times n$ unknown parameters $C_{1}^{(i)}, C_{2}^{(i)}, \forall i \in\{1,2, \ldots, n\}$.

In order to solve the first algebraic system (i), it is easy to determine the coefficients $A_{1}^{(i)}, A_{2}^{(i)} \forall i \in\{2,3, \ldots, n\}$, as function of the two coefficients of the first phase: $A_{1}^{(1)}, A_{2}^{(1)}$.

Thus, by solving the algebraic system (25), the following relations can be obtained:

$$
\begin{aligned}
& A_{1}^{(i)}=A_{1}^{(1)}\left(\frac{k^{(1)}}{k^{(i)}}\right) ; \\
& A_{2}^{(i)}=A_{2}^{(1)}+A_{1}^{(1)} k^{(1)} \sum_{j=1}^{i-1} \log R^{(j)}\left(\frac{1}{k^{(j)}}-\frac{1}{k^{(j+1)}}\right) ; \\
& \forall i \in\{2,3, \ldots, n\} .
\end{aligned}
$$

By substituting (28) into the algebraic system (26), setting $i$ equal to $n$, the constants $A_{1}^{(1)}$ and $A_{2}^{(1)}$ can be then determined, as reported below:

$$
\begin{aligned}
& A_{1}^{(1)}=\frac{T_{\mathrm{ext}}-T_{\mathrm{int}}}{\omega \log R^{(0)}}, \\
& A_{2}^{(1)}=T_{\mathrm{int}}-\frac{T_{\mathrm{ext}}-T_{\mathrm{int}}}{\omega},
\end{aligned}
$$

where $\omega=\left(k^{(1)} / \log R^{(0)}\right)\left[\sum_{j=1}^{n} \log \left(\sqrt[k^{(j)}]{R^{(j)} / R^{(j-1)}}\right)\right]$ is a nondimensional constant parameter.

Finally, the solution in closed-form of the algebraic system (i) is written as follows:

$$
\begin{aligned}
A_{1}^{(i)} & =\frac{\left(T_{\mathrm{ext}}-T_{\mathrm{int}}\right)}{k^{(i)}}\left[\frac{1}{\log \left(\prod_{j=1}^{n} \sqrt[k^{(j)}]{R^{(j)} / R^{(j-1)}}\right)}\right], \\
A_{2}^{(i)} & =T_{\mathrm{int}}+\left(T_{\mathrm{ext}}-T_{\mathrm{int}}\right) \\
& \left\{\frac{\log \left[\left(\prod_{j=1}^{i} \sqrt[k^{(j)}]{R^{(j)} / R^{(j-1)}}\right) / \sqrt[k^{(i)}]{R^{(i)}}\right]}{\log \left(\prod_{j=1}^{n} \sqrt[k^{(j)}]{R^{(j)} / R^{(j-1)}}\right)}\right\},
\end{aligned}
$$

and the temperature function in each phase can be explicitly found as

$$
\begin{aligned}
& T^{(i)}(r)=T_{\mathrm{int}}+\left(T_{\mathrm{ext}}-T_{\mathrm{int}}\right) \\
& \cdot\left\{\frac{\log \left[\left(\sqrt[k^{(i)}]{r / R^{(i)}}\right) \prod_{j=1}^{i} \sqrt[k^{(j)}]{R^{(j)} / R^{(j-1)}}\right]}{\log \left(\prod_{j=1}^{n} \sqrt[k^{(j)}]{R^{(j)} / R^{(j-1)}}\right)}\right\}, \\
& \forall i \in\{1,2, \ldots, n\} .
\end{aligned}
$$

In order to solve the second algebraic system (ii), the subsystem constituted by the equilibrium and compatibility conditions (22)-(23) is considered:

$$
\mathbf{Q} \cdot \mathbf{X}=\mathbf{L}
$$

where the vectors $\mathbf{L}=\left[\mathbf{L}^{(1)}, \mathbf{L}^{(2)}, \ldots, \mathbf{L}^{(n)}\right]^{T}$ are characterized by the following subvectors:

$$
\begin{aligned}
\mathbf{L}^{(i)}= & \boldsymbol{\Gamma}^{(i)} \mathbf{A}^{(i)}-\Gamma^{(i+1)} \mathbf{A}^{(i+1)} \\
+\left[0,\left(\Omega^{(i+1)}-\Omega^{(i)}\right)\right]^{T} T_{R}, & \\
& \forall i \in\{1,2, \ldots, n-1\},
\end{aligned}
$$

in which the vector $\mathbf{A}^{(i)}$ and the matrix $\Gamma^{(i)}$ are given by

$$
\begin{aligned}
\boldsymbol{\Gamma}^{(i)} & =\left[\begin{array}{cc}
\zeta^{(i)}\left(\log R^{(i)}-\frac{1}{2}\right) & \zeta^{(i)} \\
\psi^{(i)}\left(\log R^{(i)}-\frac{1}{2}\right) & \psi^{(i)}
\end{array}\right], \\
\mathbf{A}^{(i)} & =\left[\begin{array}{ll}
A_{1}^{(i)}, & A_{2}^{(i)}
\end{array}\right]^{T},
\end{aligned}
$$

$$
\forall i \in\{1,2, \ldots, n\}
$$

and $\mathbf{X}=\left[\mathbf{X}^{(1)}, \mathbf{X}^{(2)}, \ldots, \mathbf{X}^{(n)}\right]^{T}$ collects the unknowns subvectors $\mathbf{X}^{(i)}$, as reported below:

$$
\mathbf{X}^{(i)}=\left[C_{1}^{(i)}, C_{2}^{(i)}\right]^{T}, \quad \forall i \in\{1,2, \ldots, n\}
$$

The matrix $\mathbf{Q}$ results are then composed of the following submatrices:

$$
\begin{aligned}
& \mathbf{Q} \\
& =\left[\begin{array}{cccccccc}
-\mathbf{B}_{1}^{(1)} & \mathbf{B}_{1}^{(2)} & \mathbf{0} & \mathbf{0} & \cdots & \mathbf{0} & \mathbf{0} & \mathbf{0} \\
\mathbf{0} & -\mathbf{B}_{2}^{(2)} & \mathbf{B}_{2}^{(3)} & \mathbf{0} & \cdots & \mathbf{0} & \mathbf{0} & \mathbf{0} \\
\mathbf{0} & \mathbf{0} & -\mathbf{B}_{3}^{(3)} & \mathbf{B}_{3}^{(4)} & \cdots & \mathbf{0} & \mathbf{0} & \mathbf{0} \\
\vdots & \vdots & \vdots & \vdots & \vdots & \vdots & \vdots & \vdots \\
\mathbf{0} & \mathbf{0} & \mathbf{0} & \mathbf{0} & \cdots & -\mathbf{B}_{n-2}^{(n-2)} & \mathbf{B}_{n-2}^{(n-1)} & \mathbf{0} \\
\mathbf{0} & \mathbf{0} & \mathbf{0} & \mathbf{0} & \cdots & \mathbf{0} & -\mathbf{B}_{n-1}^{(n-1)} & \mathbf{B}_{n-1}^{(n)}
\end{array}\right],
\end{aligned}
$$


where the generic matrices $\mathbf{B}_{i}^{(i)}$ and $\mathbf{B}_{i}^{(i+1)}$, given by

$$
\begin{aligned}
\mathbf{B}_{i}^{(i)} & =\left[\begin{array}{cc}
1 & R^{(i)-2} \\
\frac{E^{(i)}}{\left(1+v^{(i)}\right)\left(2 v^{(i)}-1\right)} & \frac{E^{(i)} R^{(i)-2}}{\left(1+v^{(i)}\right)}
\end{array}\right], \\
\mathbf{B}_{i}^{(i+1)} & =\left[\begin{array}{cc}
1 & R^{(i)-2} \\
\frac{E^{(i+1)}}{\left(1+v^{(i+1)}\right)\left(2 v^{(i+1)}-1\right)} & \frac{E^{(i+1)} R^{(i)-2}}{\left(1+v^{(i+1)}\right)}
\end{array}\right],
\end{aligned}
$$

are $(2 \times 2)$ matrices with nonzero determinants, whose components have been already given above. The determinant of the matrices (37) is in particular written as follows:

$$
\begin{aligned}
\operatorname{det}\left[\mathbf{B}_{i}^{(i)}\right] & =\frac{2 E^{(i)}\left(1-v^{(i)}\right)}{R^{(i)}\left(1+\nu^{(i)}\right)\left(1-2 \nu^{(i)}\right)} \neq 0, \\
\operatorname{det}\left[\mathbf{B}_{i}^{(i+1)}\right] & =\frac{2 E^{(i+1)}\left(1-v^{(i+1)}\right)}{R^{(i)}\left(1+\nu^{(i+1)}\right)\left(1-2 \nu^{(i+1)}\right)} \neq 0 .
\end{aligned}
$$

However, in force of the special form of the matrix $\mathbf{Q}$, the algebraic system (32) can be reorganized as

$$
\begin{gathered}
\mathbf{B}_{1}^{(2)} \mathbf{X}^{(2)}=\mathbf{B}_{1}^{(1)} \mathbf{X}^{(1)}+\mathbf{L}^{(1)} \\
\mathbf{B}_{2}^{(3)} \mathbf{X}^{(3)}=\mathbf{B}_{2}^{(2)} \mathbf{X}^{(2)}+\mathbf{L}^{(2)} \\
\vdots \\
\mathbf{B}_{i}^{(i+1)} \mathbf{X}^{(i+1)}=\mathbf{B}_{i}^{(i)} \mathbf{X}^{(i)}+\mathbf{L}^{(i)} \\
\vdots \\
\mathbf{B}_{n-2}^{(n-1)} \mathbf{X}^{(n-1)}=\mathbf{B}_{n-2}^{(n-2)} \mathbf{X}^{(n-2)}+\mathbf{L}^{(n-2)} \\
\mathbf{B}_{n-1}^{(n)} \mathbf{X}^{(n)}=\mathbf{B}_{n-1}^{(n-1)} \mathbf{X}^{(n-1)}+\mathbf{L}^{(n-1)}
\end{gathered}
$$

and, by applying an in-cascade procedure, it can be finally obtained

$$
\mathbf{X}^{(i)}=\boldsymbol{\Phi}^{(i)} \mathbf{X}^{(1)}+\Psi^{(i)}, \quad \forall i \in\{2,3, \ldots, n\},
$$

where $\boldsymbol{\Phi}^{(i)}$ and $\Psi^{(i)}$ are matrix and vector, respectively. The explicit expressions of $\boldsymbol{\Phi}^{(i)}$ and $\Psi^{(i)}$ are given below:

$$
\begin{aligned}
\boldsymbol{\Phi}^{(i)} & =\prod_{j=1}^{i}\left(\left[\mathbf{B}_{i-j}^{(i-j+1)}\right]^{-1} \mathbf{B}_{i-j}^{(i-j)}\right), \\
\boldsymbol{\Psi}^{(i)} & =\mathbf{B}_{i-1}^{(i)}\left\{\mathbf{L}^{(i-1)}\right. \\
& \left.+\sum_{j=1}^{i-2}\left[\prod_{k=1}^{j}\left(\mathbf{B}_{i-k}^{(i-k)}\left[\mathbf{B}_{i-k-1}^{(i-k)}\right]^{-1}\right)\right] \mathbf{L}^{(i-j-1)}\right\},
\end{aligned}
$$

$$
\forall i \in\{2,3, \ldots, n\} \text {. }
$$

By means of (40) and (41), the generic unknowns subvector $\mathbf{X}^{(i)}$ can be written as a function of the transferring matrix $\Phi^{(i)}$, the vector $\Psi^{(i)}$, and the unknowns subvector $\mathbf{X}^{(1)}$. By imposing the two boundary conditions described by the equations obtained above, the differential problem reduces to an algebraic one in which only two coefficients, collected in $\mathbf{X}^{(1)}$ and related to the first phase, have to be determined. Therefore, in order to find the two unknowns collected in $\mathbf{X}^{(1)}$, the boundary conditions (24) can be written in matrix form. In particular, by applying (40) for $i=n$, the following relationship is obtained:

$$
\mathbf{X}^{(n)}=\boldsymbol{\Phi}^{(n)} \mathbf{X}^{(1)}+\Psi^{(n)}
$$

Then, the boundary conditions (24) become

$$
\mathbf{P X}^{(1)}=\mathbf{L}_{P}
$$

where the matrix $\mathbf{P}$ and the vector $\mathbf{L}_{P}$ are given by

$$
\begin{aligned}
\mathbf{P} & =\left[\begin{array}{c}
\mathbf{P}_{1} \\
{\left[\boldsymbol{\Phi}^{(n)}\right]^{T} \mathbf{P}_{2}}
\end{array}\right], \\
\mathbf{L}_{P} & =\left[\begin{array}{c}
L_{1 P} \\
L_{2 P}-\mathbf{P}_{2} \cdot \Psi^{(n)}
\end{array}\right],
\end{aligned}
$$

$\mathbf{P}_{1}$ and $\mathbf{P}_{2}$ being two vectors, dot denoting the standard scalar product, and $L_{1 P}, L_{2 P}$ being two scalars, whose explicit expressions are

$$
\begin{aligned}
\mathbf{P}_{1}= & \frac{E^{(1)}}{\left(1+\nu^{(1)}\right)}\left[\frac{1}{\left(1-2 \nu^{(1)}\right)},-R^{(0)-2}\right]^{T}, \\
\mathbf{P}_{2}= & \frac{E^{(n)}}{\left(1+\nu^{(n)}\right)}\left[\frac{1}{\left(1-2 \nu^{(n)}\right)},-R^{(n)-2}\right]^{T}, \\
L_{1 P}= & -\Omega^{(1)} T_{R}+\psi^{(1)}\left[A_{1}^{(1)}\left(\log R^{(0)}-\frac{1}{2}\right)+A_{2}^{(1)}\right] \\
& +p_{i}, \\
L_{2 p}= & -\Omega^{(n)} T_{R}+\psi^{(n)}\left[A_{1}^{(n)}\left(\log R^{(n)}-\frac{1}{2}\right)+A_{2}^{(n)}\right] \\
& +p_{e} .
\end{aligned}
$$

Therefore, by inverting all the $2 \times n$ unknown coefficients, the unknown parameters are finally found out:

$$
\begin{aligned}
& \mathbf{X}^{(1)}=\mathbf{P}^{-1} \mathbf{L}_{P}, \\
& \mathbf{X}^{(i)}=\boldsymbol{\Phi}^{(i)}\left(\mathbf{P}^{-1} \mathbf{L}_{P}\right)+\Psi^{(i)}, \quad \forall i \in\{2,3, \ldots, n\} .
\end{aligned}
$$

By substituting the relationships (46) and (30) in (17), the explicit analytical expression of the solution in terms of displacements for each phase of the multilayer hollow cylinder can be obtained. 


\section{Radius and Thickness for Insulated Pipelines according to European Standards EN 253}

Generally, the industrial insulate pipeline is composed of three phases: steel pipe, insulate coating, and external casing. The minimum dimensions of thickness of the steel pipe and casing are prescribed in European Standards EN 253, as function of nominal outside diameter. By fitting the values given by EN 253, the following expression describes the minimum values of the thickness of the steel pipe, as function of the inner radius

$$
\delta^{(1)}=\left(3+16.5 R^{(0)}\right) 10^{-3}[\mathrm{~m}] .
$$

Similarly, the minimum value of the thickness $\delta^{(3)}$ of the casing, as function of maximum radius of the casing $R^{(2)}$, is reported below:

$$
\delta^{(3)}=\left(2.5+14.6 R^{(2)}\right) 10^{-3}[\mathrm{~m}] .
$$

By applying the relationships (47) and (48), the geometrical parameters of the insulate pipeline depend on two parameters $R^{(0)}$ and $\delta^{(2)}$ only, as reported below:

$$
\begin{aligned}
& R^{(1)}=3 \cdot 10^{-3}+1.0165 R^{(0)}, \\
& R^{(2)}=3 \cdot 10^{-3}+1.0165 R^{(0)}+\delta^{(2)}, \\
& R^{(3)}=5.5438 \cdot 10^{-3}+1.03134 R^{(0)}+1.0146 \delta^{(2)} .
\end{aligned}
$$

The inner radii of the steel pipe $R^{(0)}$ and insulate coating $R^{(2)}$ are usually found to vary within the following ranges:

$$
\begin{aligned}
& R^{(0)} \in\left[8.65 \cdot 10^{-3} \mathrm{~m}, 0.597 \mathrm{~m}\right], \\
& R^{(2)} \in[0.0345,0.6875 \mathrm{~m}] .
\end{aligned}
$$

By applying the relationship (49), if the inner radius $R^{(0)}$ is prescribed, the thickness $\delta^{(2)}$ must satisfy the inequalities:

$$
\begin{gathered}
0 \leq \delta^{(2)} \leq 0.078 \text { for } R^{(0)}=0.597 \\
0.0227 \leq \delta^{(2)} \leq 0.6757 \text { for } R^{(0)}=0.00865 .
\end{gathered}
$$

The domain of the parameters $R^{(0)}$ and $\delta^{(2)}$, satisfying the values given by EN 253, is reported in Figure 2.

\section{Parametric Analysis of an Industrial Insulated Pipeline Composed of Three Phases}

In this section, an industrial insulated pipeline has been modelled by means of a multilayered solid composed of three hollow cylinders, as showed in Figure 1(b). The materials characterizing the insulated pipeline are steel (phase 1), insulated coating (phase 2), and polyethylene (phase 3), adopted to protect the insulation. Accordingly to European Standards,

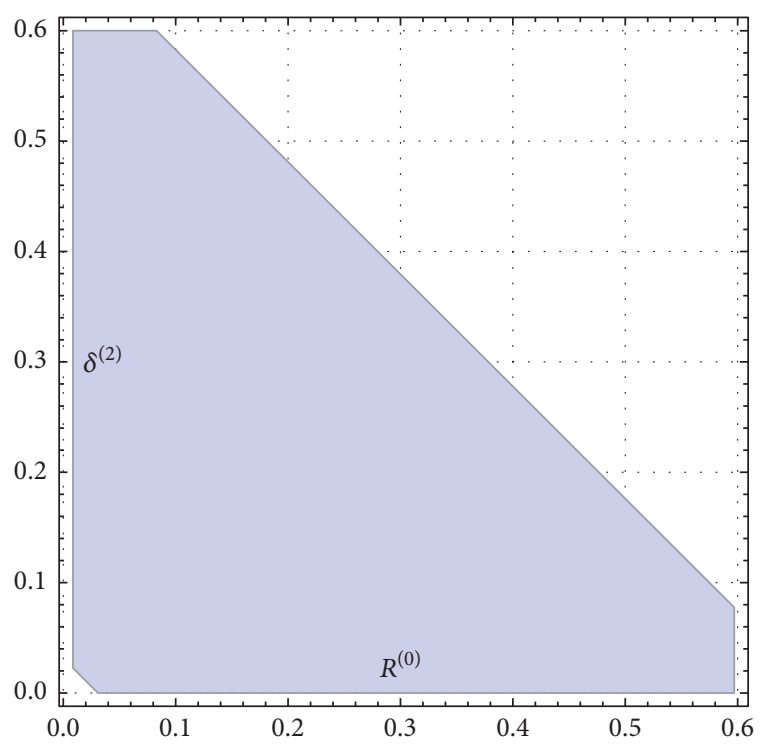

FIGURE 2: Domain of the parameters $R^{(0)}$ and $\delta^{(2)}$.

TABLE 1: Mechanical and thermal parameters of steel and polyethylene.

\begin{tabular}{lcc}
\hline & Steel pipe & Polyethylene jacket \\
\hline$E\left[\mathrm{~N} \mathrm{~m}^{-2}\right]$ & $210 \cdot 10^{9}$ & $0.7 \cdot 10^{9}$ \\
$k\left[\mathrm{~W} \mathrm{~m}^{-1} \mathrm{~K}^{-1}\right]$ & 45 & 0.40 \\
$\nu$ & 0.30 & 0.42 \\
$\alpha\left[\mathrm{m} \cdot \mathrm{m}^{-1} \mathrm{~K}^{1}\right]$ & $1.2 \cdot 10^{-5}$ & $2.25 \cdot 10^{-4}$ \\
\hline
\end{tabular}

the inner radius and the thickness of the pipeline and the polyethylene jacket are fixed by applying the limitation reported in the EN 253.

The inner radius and thickness of the pipeline and the polyethylene jacket have been set to $R_{0}=0.20 \mathrm{~m}$ and $\delta^{(2)}=$ $0.20 \mathrm{~m}$, for the case at hand. By applying the relationships (47), (48), and (49), the other geometrical parameters of the multilayered cylinder have been obtained and detailed below:

$$
\begin{aligned}
& \delta^{(1)}=6.3 \cdot 10^{-3}[\mathrm{~m}], \\
& \delta^{(3)}=8.432 \cdot 10^{-3}[\mathrm{~m}], \\
& R^{(1)}=0.206[\mathrm{~m}], \\
& R^{(2)}=0.406[\mathrm{~m}], \\
& R^{(3)}=0.415[\mathrm{~m}] .
\end{aligned}
$$

Also, mechanical and thermal properties of steel and polyethylene are reported in Table 1.

Parametrical analyses have been thus conducted by considering two loading conditions with different subcases:

(a) Pipeline subject to temperature gradient between the internal and external surfaces $\Delta T=T_{\text {int }}-T_{\text {ext }}$.

(b) Pipeline subject to internal pressure, with parametrically varying Poisson's ratios and Young's moduli. 


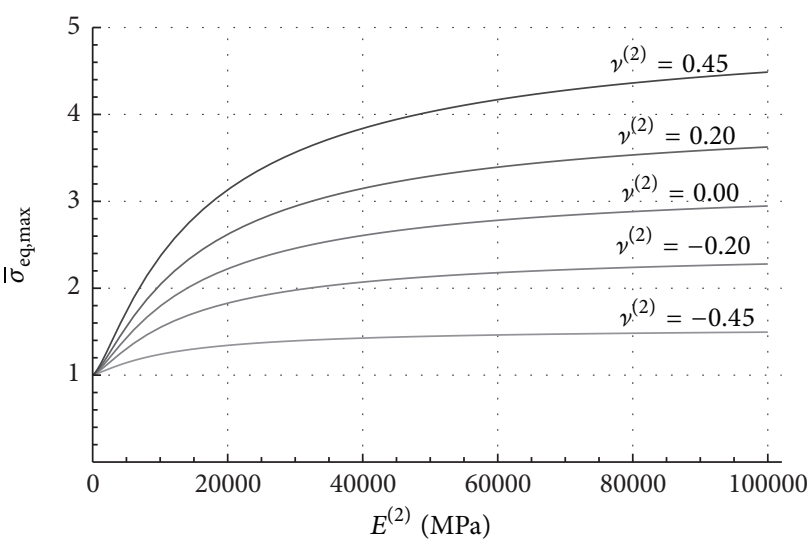

(a)

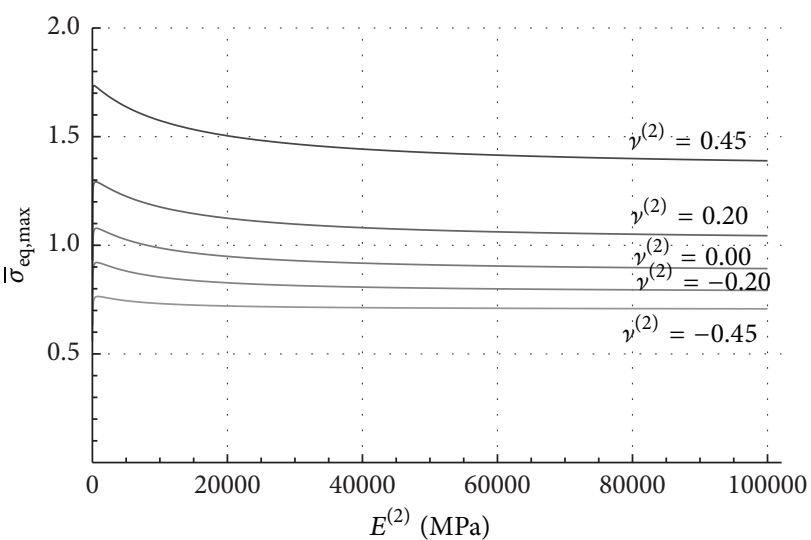

(b)

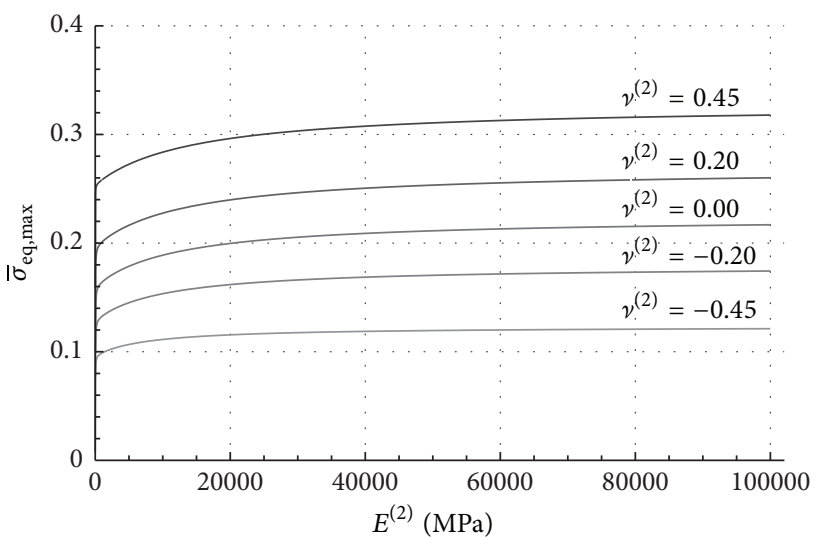

(c)

FIGURE 3: Maximum value of nondimensional equivalent stress in the steel (phase 1: (a)), in the insulated coating (phase 2: (b)), and in polyethylene jacket (phase 3: (c)) in the case of insulate pipeline subjected to temperature gradient.

In particular, two further subscenarios have been analyzed for case (a):

(i) Poisson's ratio and Young's modulus have been taken as varying, with fixed values of both thermal conductivity and linear thermal expansion coefficients.

(ii) Young's moduli and linear thermal expansion coefficient have been parametrically varied, prescribing both Poisson's ratio and the thermal conductivity coefficients.

In order to localize the onset of plasticity, in the case of planar isotropy and nonhomogeneity in radial direction, the equivalent stress based on the Hencky-von Mises criterion has been chosen, in the form recalled below:

$$
\begin{aligned}
\sigma_{\mathrm{eq}}^{(i)} & =2^{-1 / 2}\left[\left(\sigma_{r r}^{(i)}-\sigma_{\theta \theta}^{(i)}\right)^{2}+\left(\sigma_{r r}^{(i)}-\sigma_{z z}^{(i)}\right)^{2}\right. \\
& \left.+\left(\sigma_{\theta \theta}^{(i)}-\sigma_{z z}^{(i)}\right)^{2}\right]^{1 / 2} .
\end{aligned}
$$

The following dimensionless parameters for the maximum absolute value of nondimensional equivalent stress have been also taken into consideration:

$$
\begin{aligned}
& \bar{\sigma}_{\text {eq }}^{(i)}=\frac{\sigma_{\text {eq }}^{(i)}}{\alpha^{(i)} E^{(i)}\left(T_{i}-T_{e}\right)}, \text { for } p_{\text {int }}=0, T_{i}>T_{e}=T_{R}, \\
& \bar{\sigma}_{\text {eq }}^{(i)}=\frac{\sigma_{\text {eq }}^{(i)}}{p_{\text {int }}}, \text { for } p_{\text {int }} \neq 0, T_{e}=T_{i}=T_{R} .
\end{aligned}
$$

The effects of Poisson ratio and Young modulus variation on the maximum value of nondimensional equivalent stress are shown in Figure 3, when an insulated pipeline is subject to thermal gradient, load case (a), in the steel (phase 1), in the insulated coating (phase 2), and in the polyethylene jacket (phase 3).

It is worth noticing that, by increasing Young's modulus of the insulate coating, the maximum values of the equivalent stress increase in the steel pipe (Figure 3(a)), while, by fixing the value of the Poisson ratio, the maximum values of the equivalent stress in both insulate coating (Figure 3(b)) and polyethylene jacket (Figure 3(a)) tend to be not significantly 


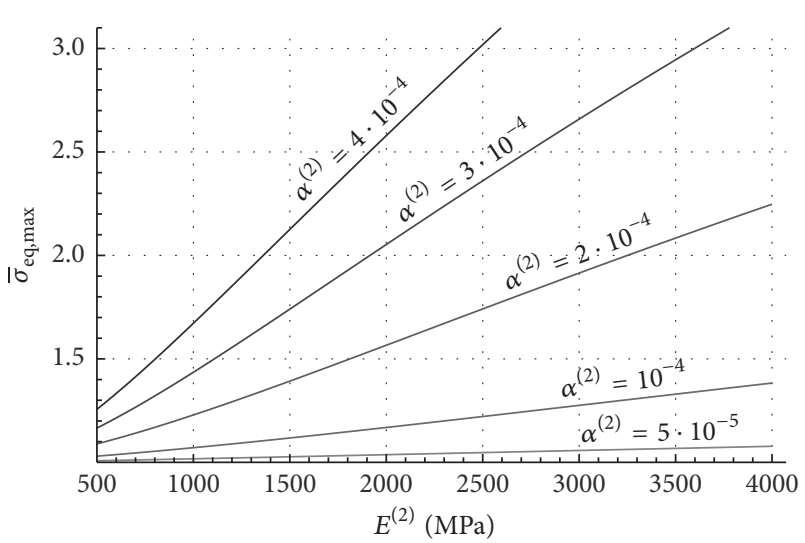

(a)

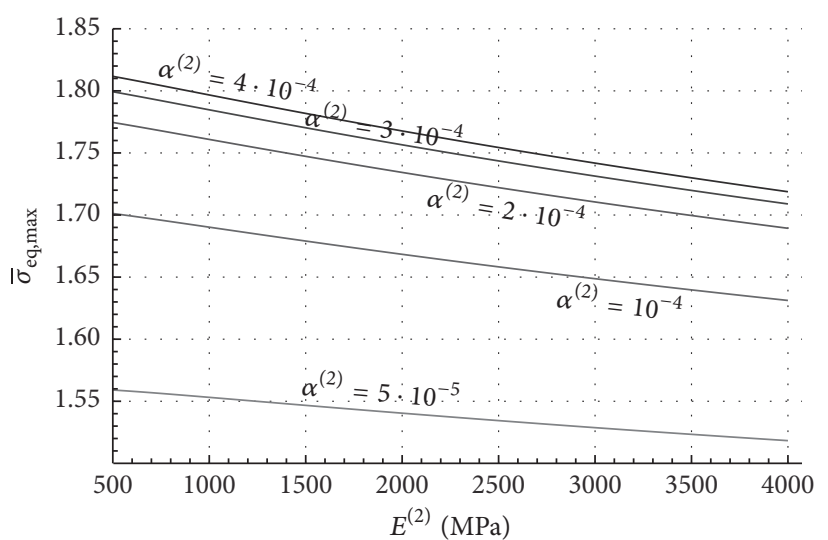

(b)

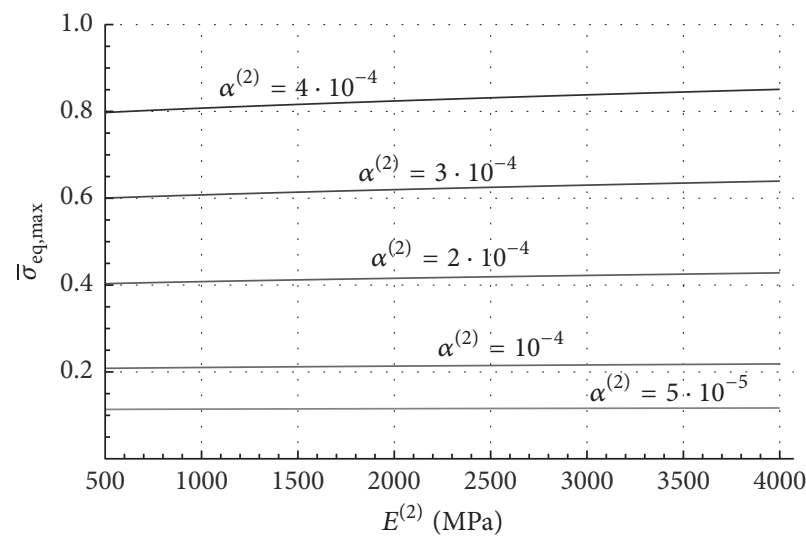

(c)

Figure 4: Maximum value of nondimensional equivalent stress in the steel (phase 1: (a)), in the insulated coating (phase 2: (b)), and in polyethylene jacket (phase 3: (c)) in the case of insulate pipeline subjected to temperature gradient.

affected by the Young moduli as they increase. Moreover, higher Poisson's ratios of insulate coating increase the maximum values of the equivalent stress in each phase and, in the particular case in which the insulated coating is composed of auxetic material with negative Poisson's ratio, the maximum equivalent stress significantly reduces in each phase.

Similarly, the results, in terms of the maximum values of the equivalent stress in each phase, obtained by varying the Young modulus and the linear thermal expansion coefficient, are shown in Figure 4.

At fixed value of the thermal expansion coefficient, by increasing Young's modulus of the insulate coating, the equivalent stress increases in the steel pipe and remains practically constant in both the polyethylene jacket and the insulate coating. Moreover, by increasing the linear thermal expansion coefficient of the insulate coating, the maximum values of the equivalent stress increase in each phase.

By considering the load case (b), a parametric analysis has been instead conducted with the aim of studying the effects of Poisson's ratio and Young's modulus variation on the mechanical response of an insulated pipeline subject to internal radial pressure. In particular, the maximum values of nondimensional equivalent stress in the steel, in the insulated coating and in the polyethylene jacket, are shown in Figure 5.
By increasing Young's modulus in the insulate coating, the maximum equivalent stress decreases in the steel pipe while, by decreasing Poisson's ratio in the insulate coating, the maximum values of the equivalent stress decrease in the steel pipe up to reach lowest values as Poisson's ratios tend to negative (auxetic) values (Figure 5(a)). A complementary behaviour is instead observed in the insulated coating phase (Figure 5(b)).

Finally, by increasing Young's modulus in the insulate coating, the maximum equivalent stress decreases in the polyethylene jacket while, by decreasing Poisson's ratio in the insulate coating, the maximum values of the equivalent stress increase in the polyethylene jacket (Figure 5(c)).

\section{Numerical Example of an Insulated Pipeline Subject to Temperature Gradient}

In the present section, a numerical example, an insulated pipeline subject to temperature gradient (jump) equal to $120^{\circ} \mathrm{C}$ and constituted by three phases (steel pipe, insulate coating, and polyethylene jacket), is reported. The nondimensional equivalent stress, calculated by applying formulas (54), has been evaluated for three types of materials characterizing the insulate coating: (1) expanded polyurethane, (2) laminate 


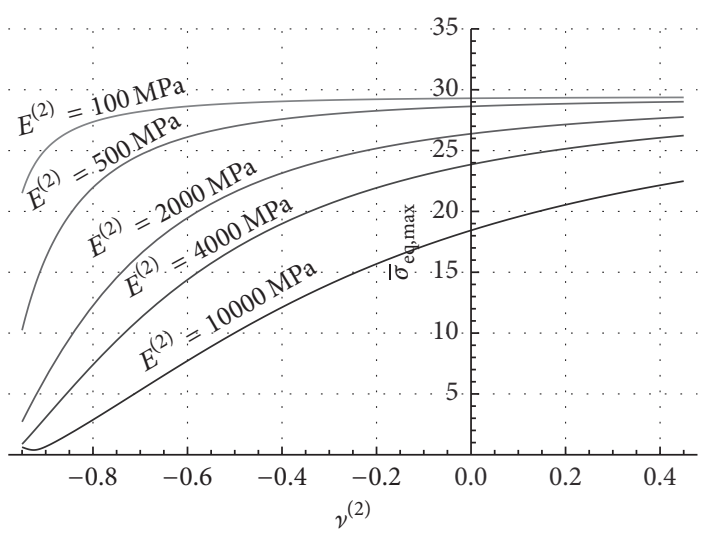

(a)

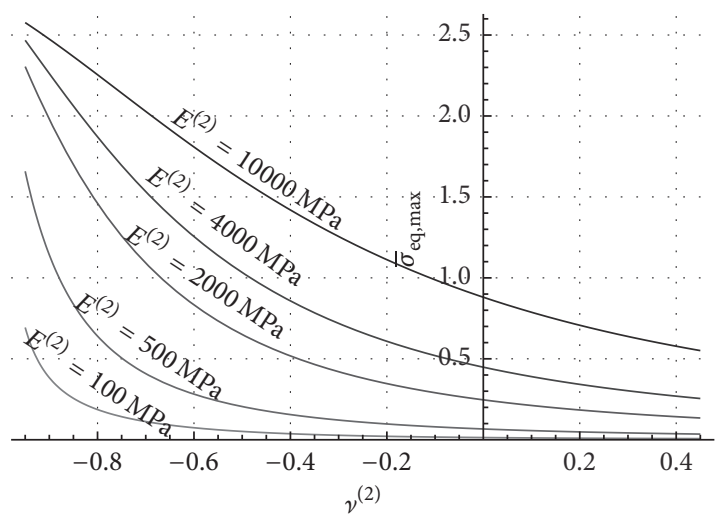

(b)

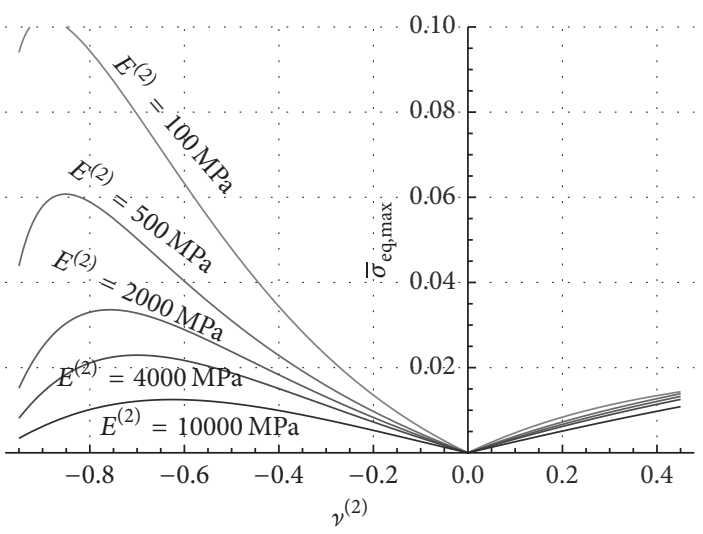

(c)

FIGURE 5: Maximum value of nondimensional equivalent stress in the steel (phase 1: (a)), in the insulated coating (phase 2: (b)), and in polyethylene jacket (phase 3: (c)) in the case of insulate pipeline subjected to internal radial pressure.

glass, and (3) syntactic foam. The mechanical and thermal properties of the adopted materials are reported in detail in Table 2.

By selecting the steel pipe and the polyethylene jacket with nominal diameter equal to $400 \mathrm{~mm}$ and $800 \mathrm{~mm}$, respectively, the values of the inner radius and the thickness of phases (1) and (3) have been determined, accordingly to already cited rule EN 253:

$$
\begin{aligned}
\text { DN400 (steel) } & \longrightarrow R^{(0)}=0.197[\mathrm{~m}] ; \\
\delta^{(1)} & =6.249 \cdot 10^{-3}[\mathrm{~m}], \\
\text { DN800 (jacket) } & \longrightarrow R^{(2)}=0.396[\mathrm{~m}] ; \\
\delta^{(3)} & =8.283 \cdot 10^{-3}[\mathrm{~m}] .
\end{aligned}
$$

The other geometrical parameters have been thus calculated as follows:

$$
\begin{aligned}
& R^{(1)}=R^{(0)}+\delta^{(1)}=0.203[\mathrm{~m}] ; \\
& R^{(3)}=R^{(2)}+\delta^{(3)}=0.404[\mathrm{~m}] ; \\
& \delta^{(2)}=R^{(2)}-R^{(0)}-\delta^{(1)}=0.193[\mathrm{~m}] ;
\end{aligned}
$$

Figure 6 shows the equivalent stress versus the pipeline radius.
Along the steel pipe phase radius (Figure 5(a)), if the insulate coating is constituted by laminate glass (Case 2 ), the nondimensional equivalent stress assumes values smaller than the other cases (Cases 1 and 3), due to the fact that the linear thermal expansion coefficient of laminate glass is smaller than those of expanded polyurethane and syntactic foam. On the other hand, along the insulate coating phase radius (Figure 5(b)), if the insulate coating is constituted by expanded polyurethane (Case 1), the nondimensional equivalent stress assumes values greater than the other cases (Cases 2 and 3) because Young's modulus of expanded polyurethane is smaller than those of laminate glass and syntactic foam, while, moving along the polyethylene jacket radius (Figure 5(c)), if the insulate coating is constituted by laminate glass (Case 2), the nondimensional equivalent stress assumes values smaller than the other cases (Cases 1 and 3) because the linear thermal expansion coefficient of laminate glass is smaller than those of expanded polyurethane and syntactic foam.

\section{Conclusions}

In the present work, analytical solutions and numerical results for a class of multilayered cylinders under combined thermomechanical conditions have been obtained. 


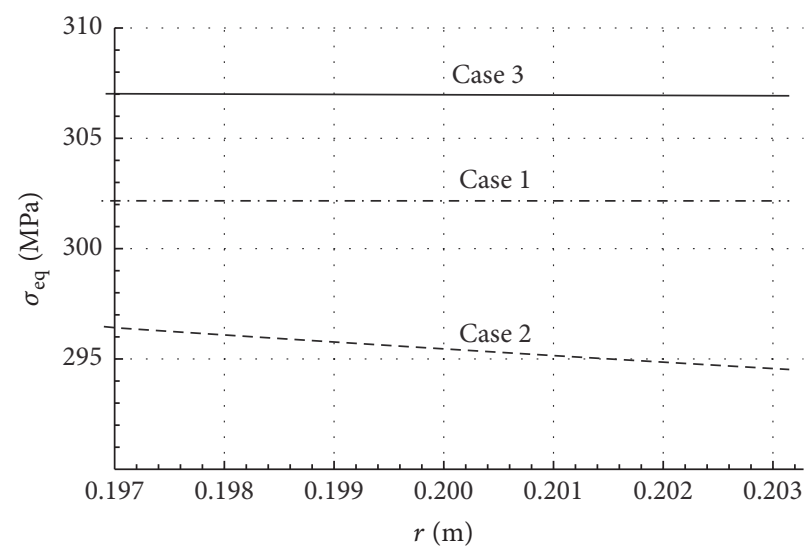

(a)

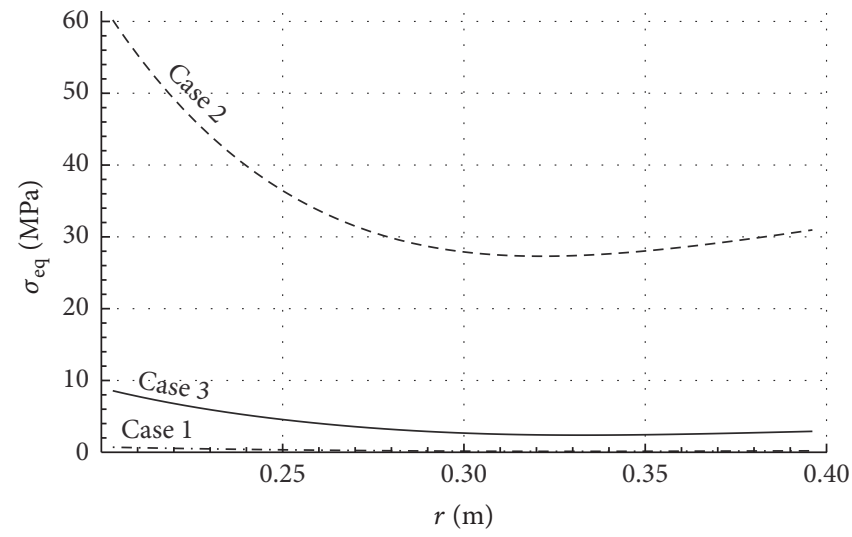

(b)

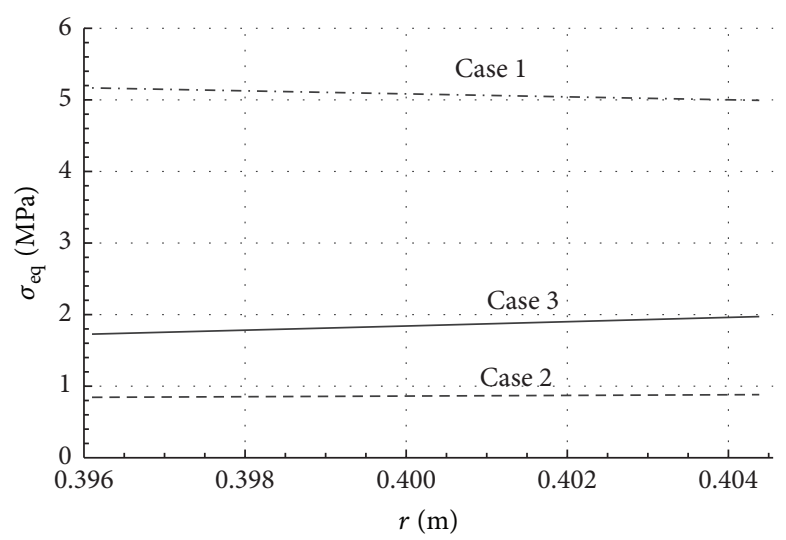

(c)

FIGURE 6: Equivalent stress [MPa] along the radius in the case of insulate pipeline subjected to temperature gradient in the steel (a), in the insulate coating (b), and in the polyethylene jacket (c). Case 1: expanded polyurethane, Case 2: laminate glass, and Case 3: syntactic foam.

TABLE 2: Mechanical and thermal parameters of the following materials: (1) expanded polyurethane, (2) laminate glass, and (3) syntactic foam.

\begin{tabular}{lccc}
\hline & Expanded polyurethane & Laminate glass & Syntactic foam \\
\hline$E\left[\mathrm{~N} \mathrm{~m}^{-2}\right]$ & $0.025 \cdot 10^{9}$ & $26 \cdot 10^{9}$ & $1.10 \cdot 10^{9}$ \\
$k\left[\mathrm{~W} \mathrm{~m}^{-1} \mathrm{~K}^{-1}\right]$ & 0.017 & 0.036 & 0.165 \\
$v$ & 0.49 & 0.28 & 0.32 \\
$\alpha\left[\mathrm{m} \cdot \mathrm{m}^{-1} \mathrm{~K}^{1}\right]$ & $1.25 \cdot 10^{-4}$ & $1.9 \cdot 10^{-5}$ & $5.0 \cdot 10^{-5}$ \\
\hline
\end{tabular}

The closed-form thermoelastic solution has been in particular found first for a generic FGM hollow cylinder with $n$ layers and thus for a multilayer hollow cylinder composed of three phases, steel pipe, insulating coating, and polyethylene jacket, and subject to two prescribed loading conditions. Parametric analyses have been conducted by varying mechanical properties of the adopted materials, by making reference to the Hencky-von Mises criterion to localize the possible onset of plasticity. The results have shown that when the insulated pipeline is subject to temperature gradients, the maximum equivalent stress reduces in steel pipe, coating, and jacket, if the linear thermal expansion coefficient of insulate coating assumes values close to the linear thermal expansion coefficient of the steel, being thus possible to significantly reduce the maximum equivalent stress in the pipeline and avoid failure by utilizing the insulated coating composed of auxetic material (with negative Poisson's ratio). Moreover, when the insulate pipeline is subjected to radial pressure, insulated coating with negative Poisson's ratio shows reduced values of the maximum equivalent stress in steel phase only, but the values in the other two phases result generally increased.

Finally, a numerical example related to a case of practical engineering interest, that is, a hollow cylinder composed of three different insulation materials and thicknesses according to $\mathrm{EN} \mathrm{253,} \mathrm{has} \mathrm{been} \mathrm{presented,} \mathrm{the} \mathrm{results} \mathrm{showing} \mathrm{that}$ 
the laminate glass insulation materials induce values of the nondimensional equivalent stress smaller than the corresponding ones calculated in presence of the other materials, both in the steel pipe and in the polyethylene jacket.

Given the wide interest in the field, it is felt that the obtained results, based on ad hoc constructed analytical solutions, could be helpfully utilized with the aim of optimizing the design and predict failure phenomena of industrial insulate pipelines subject to combined mechanical and thermal loads, the proposed method of solution being in principle exploitable for a wide range of physical-engineering problems involving the thermoelastic response of functionally graded material structures.

\section{Nomenclature}

$k$ : Thermal conductivity coefficient $\left[\mathrm{W} \mathrm{m}^{-1} \mathrm{~K}^{-1}\right]$

A: Linear thermal expansion coefficient $\left[{ }^{\circ} \mathrm{K}^{-1}\right]$

$E$ : Young's modulus $[\mathrm{Pa}]$

$v$ : Poisson's ratio

$T_{\text {int }}$ : Internal surface temperature in steady-state conditions $\left[{ }^{\circ} \mathrm{K}\right]$

$T_{\text {ext }}$ : External surface temperature in steady-state conditions $\left[{ }^{\circ} \mathrm{K}\right]$

$p_{\text {int }}:$ Internal surface pressure $[\mathrm{Pa}]$

$p_{\text {ext }}$ : External surface pressure $[\mathrm{Pa}]$

T: $\quad$ Temperature $\left[{ }^{\circ} \mathrm{K}\right]$

$r$ : Radial coordinate $[\mathrm{m}]$

$u_{r}^{(i)}$ : Radial displacement in the generic $i$ th phase $[\mathrm{m}]$

$\varepsilon_{j k}^{(i)}$ : Strain components in the generic $i$ th phase

$\sigma_{j k}^{(i)}$ : Stress components in the generic $i$ th phase $[\mathrm{Pa}]$.

\section{Competing Interests}

The authors declare that they have no competing interests.

\section{References}

[1] B. A. Boley and J. H. Weiner, Theory of Thermal Stresses, Dover, Mineola, NY, USA, 1997.

[2] F. Alavi, D. Karimi, and A. Bagri, "An investigation on thermoelastic behaviour of functionally graded thick spherical vessels under combined thermal and mechanical loads," Journal of Achievements in Materials and Manufacturing Engineering, vol. 31, no. 2, pp. 422-428, 2008.

[3] A. N. Eraslan and T. Akis, "Plane strain analytical solutions for a functionally graded elastic-plastic pressurized tube," International Journal of Pressure Vessels and Piping, vol. 83, no. 9, pp. 635-644, 2006.

[4] Y. Obata and N. Noda, "Steady thermal stresses in a hollow circular cylinder and a hollow sphere of a functionally gradient material," Journal of Thermal Stresses, vol. 17, no. 3, pp. 471-487, 1994.

[5] Y. Ootao and Y. Tanigawa, "Three-dimensional transient thermal stresses of functionally graded rectangular plate due to partial heating," Journal of Thermal Stresses, vol. 22, no. 1, pp. 35-55, 1999.

[6] K.-S. Kim and N. Noda, "Green's function approach to unsteady thermal stresses in an infinite hollow cylinder of functionally graded material," Acta Mechanica, vol. 156, no. 3-4, pp. 145-161, 2002.

[7] M. Jabbari, S. Sohrabpour, and M. R. Eslami, "Mechanical and thermal stresses in a functionally graded hollow cylinder due to radially symmetric loads," International Journal of Pressure Vessels and Piping, vol. 79, no. 7, pp. 493-497, 2002.

[8] A. N. Eraslan, "Thermally induced deformations of composite tubes subjected to a nonuniform heat source," Journal of Thermal Stresses, vol. 26, no. 2, pp. 167-193, 2003.

[9] Z. S. Shao, "Mechanical and thermal stresses of a functionally graded circular hollow cylinder with finite length," International Journal of Pressure Vessels and Piping, vol. 82, no. 3, pp. 155-163, 2005.

[10] M. Fraldi, F. Carannante, and L. Nunziante, "Analytical solutions for $\mathrm{n}$-phase functionally graded material cylinders under de saint venant load conditions: homogenization and effects of poisson ratios on the overall stiffness," Composites Part B: Engineering, vol. 45, no. 1, pp. 1310-1324, 2013.

[11] M. Fraldi, L. Nunziante, and F. Carannante, "Axis-symmetrical solutions for n-plies functionally graded material cylinders under strain no-decaying conditions," Mechanics of Advanced Materials and Structures, vol. 14, no. 3, pp. 151-174, 2007.

[12] K. M. Liew, S. Kitipornchai, X. Z. Zhang, and C. W. Lim, "Analysis of the thermal stress behaviour of functionally graded hollow circular cylinders," International Journal of Solids and Structures, vol. 40, no. 10, pp. 2355-2380, 2003.

[13] N. Bouchonneau, V. Sauvant-Moynot, D. Choqueuse, F. Grosjean, E. Poncet, and D. Perreux, "Experimental testing and modelling of an industrial insulated pipeline for deep sea application," Journal of Petroleum Science and Engineering, vol. 73, no. 1-2, pp. 1-12, 2010.

[14] Y.-S. Choi, M.-K. Chung, and J.-G. Kim, "Effects of cyclic stress and insulation on the corrosion fatigue properties of thermally insulated pipeline," Materials Science and Engineering A, vol. 384, no. 1-2, pp. 47-56, 2004.

[15] D. Deng and H. Murakawa, "Numerical simulation of temperature field and residual stress in multi-pass welds in stainless steel pipe and comparison with experimental measurements," Computational Materials Science, vol. 37, no. 3, pp. 269-277, 2006.

[16] A. Yaghi, T. H. Hyde, A. A. Becker, W. Sun, and J. A. Williams, "Residual stress simulation in thin and thick-walled stainless steel pipe welds including pipe diameter effects," International Journal of Pressure Vessels and Piping, vol. 83, no. 11-12, pp. 864874, 2006.

[17] H. F. Nied and F. Erdogan, "Transient thermal stress problem for a circumferentially cracked hollow cylinder," NASA Contractor Report 165989, 1982.

[18] M. Nemat-Alla, K. I. E. Ahmed, and I. Hassab-Allah, "Elasticplastic analysis of two-dimensional functionally graded materials under thermal loading," International Journal of Solids and Structures, vol. 46, no. 14-15, pp. 2774-2786, 2009.

[19] T. Akis, "Elastoplastic analysis of functionally graded spherical pressure vessels," Computational Materials Science, vol. 46, no. 2, pp. 545-554, 2009. 


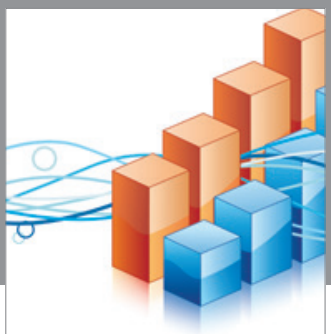

Advances in

Operations Research

vatem alat4

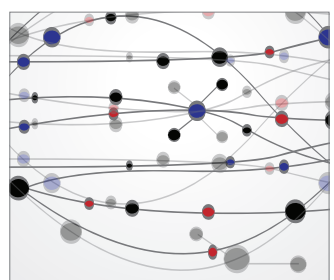

\section{The Scientific} World Journal
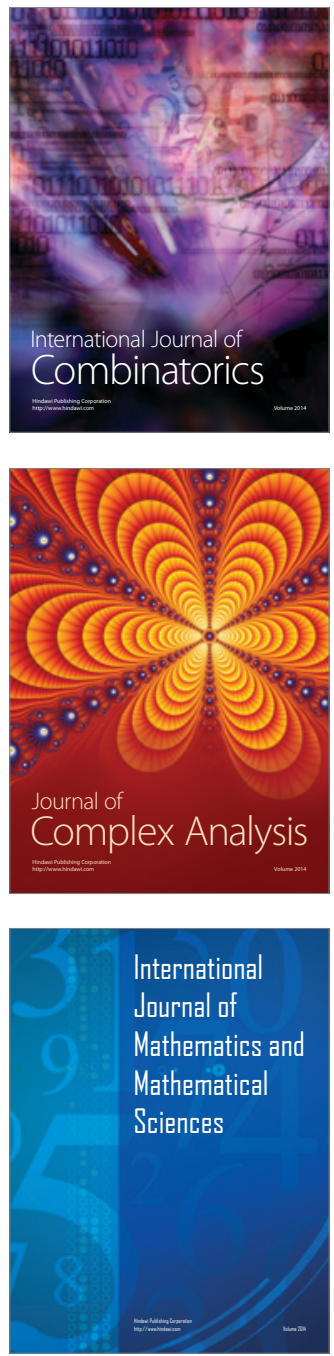
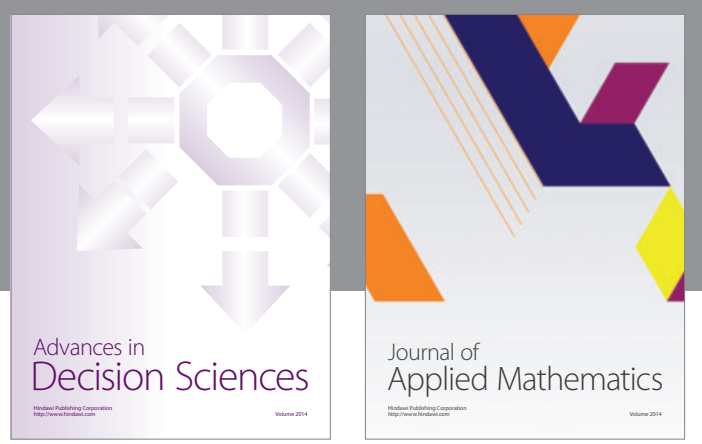

Algebra

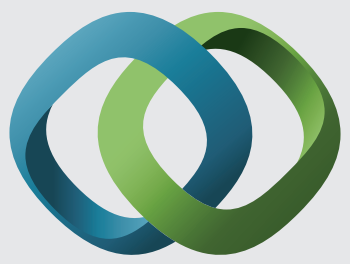

\section{Hindawi}

Submit your manuscripts at

http://www.hindawi.com
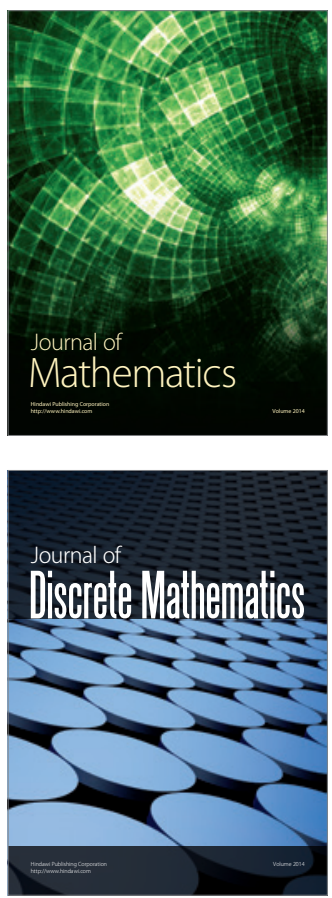

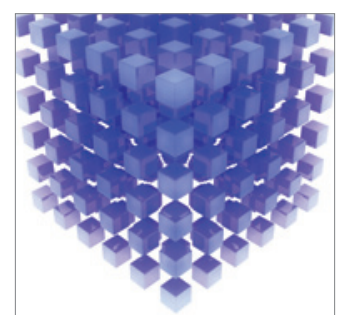

Mathematical Problems in Engineering
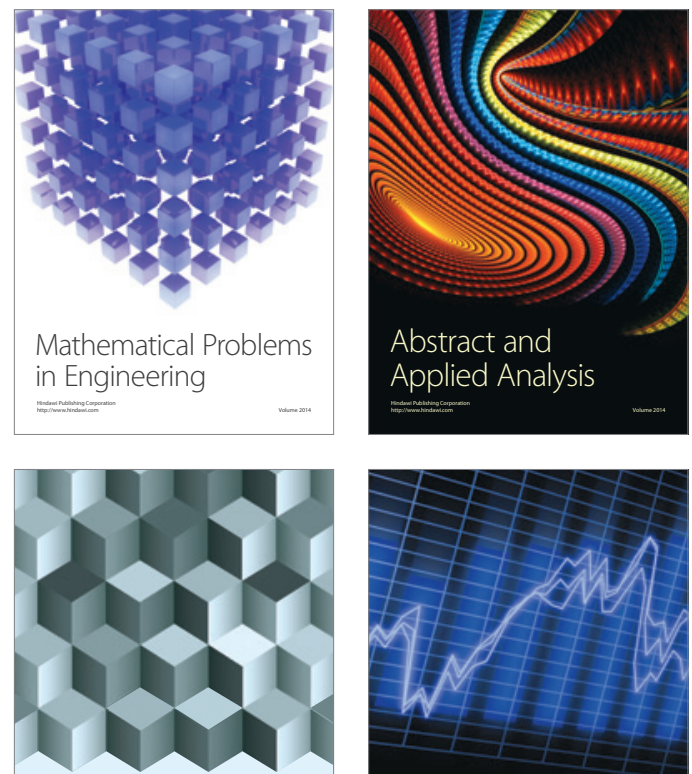

Journal of

Function Spaces

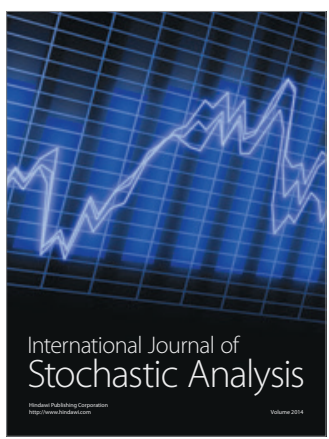

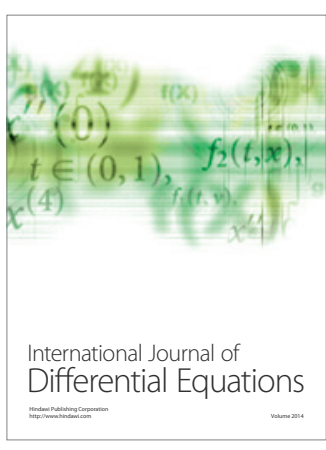
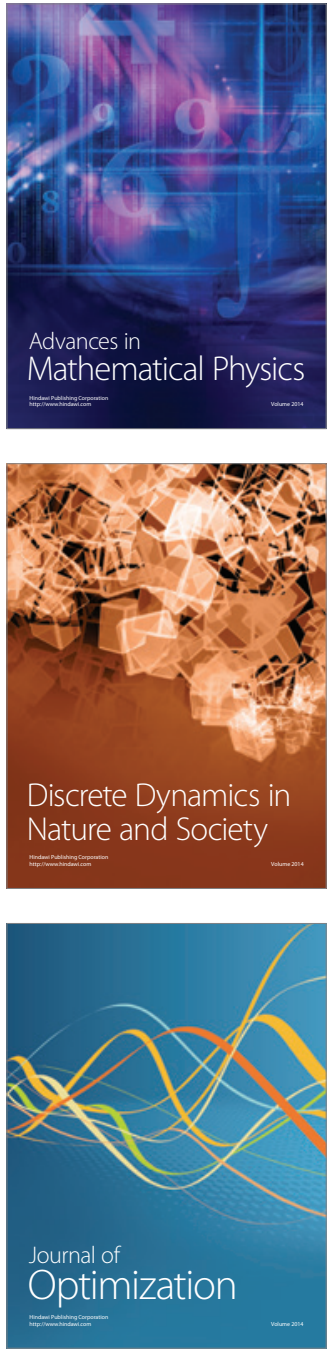